\title{
Prophylaxis with Human Serum Butyrylcholinesterase Protects Göttingen Minipigs Exposed to a Lethal High-Dose of Sarin Vapor
}

Ashima Saxena ${ }^{1,3}$, Nicholas B. Hastings ${ }^{1,4}$, Wei Sun ${ }^{1}$, Paul A. Dabisch ${ }^{2}$, Stanley W. Hulet ${ }^{2}$, Edward M. Jakubowski ${ }^{2}$, Robert J. Mioduszewski ${ }^{2}$, Bhupendra P. Doctor ${ }^{1,5}$

Division of ${ }^{1}$ Biochemistry, Walter Reed Army Institute of Research, Silver Spring, MD 20910,

${ }^{2}$ Operational Toxicology Team, Edgewood Chemical Biological Center, Aberdeen Proving Ground, MD 21010.

Present address: ${ }^{3}$ U.S. Military HIV Research Program, Walter Reed Army Institute of Research, Silver Spring, MD 20910; ${ }^{4}$ Department of Pharmacology (Neuroscience), Merck Research Laboratories, Kenilworth, NJ 07033.

${ }^{5}$ Deceased

Corresponding author:

Ashima Saxena, Ph. D.

U.S. Military HIV Research Program, Walter Reed Army Institute of Research

503 Robert Grant Ave, Silver Spring, MD 20910-7500

Phone: 301-319-9406 ; Fax: 301-319-9535

E mail:Ashima.saxena.civ@mail.mil 
Abbreviations used: ChE, cholinesterase; AChE, acetylcholinesterase; Hu BChE, human serum butyrylcholinesterase; OP, organophosphorus compounds; GB (sarin), $O$-isopropyl methylphosphonofluoridate; GD (soman), $O$-pinacolyl methylphosphonofluoridate; GA (tabun), $O$-ethyl $N, N$-dimethylphosphoramidocyanate; VX, $O$-ethyl $S$-2- $N, N$-diisopropylaminoethyl methylphosphonothiolate; CNS, central nervous system; ECG, electrocardiogram; EEG, electroencephalogram; $\mathrm{LCt}_{50}$, the product of vapor concentration and exposure time that will cause death in $50 \%$ of an exposed population. 


\begin{abstract}
Serum-derived human butyrylcholinesterase $(\mathrm{Hu} \mathrm{BChE})$ is a stoichiometric bioscavenger that is being developed as a potential prophylactic nerve agent countermeasure. Previously, we reported the prophylactic efficacy of $\mathrm{Hu} \mathrm{BChE}$ in Göttingen minipigs against a whole-body exposure to $4.1 \mathrm{mg} / \mathrm{m}^{3}$ of sarin (GB) vapor, which produced lethality over $60 \mathrm{~min}$. Since the toxicity of nerve agent is concentration-dependent, in the present study, we investigated the toxic effects of an almost 3-fold higher rate of GB vapor exposure and the ability of $\mathrm{Hu} \mathrm{BChE}$ to protect minipigs against this exposure. Male minipigs were subjected to: (1) Air exposure; (2) GB vapor exposure; or (3) pretreatment with $7.5 \mathrm{mg} / \mathrm{kg}$ of $\mathrm{Hu} \mathrm{BChE}$ by i.m. injection, $24 \mathrm{~h}$ prior to whole-body exposure to $11.4 \mathrm{mg} / \mathrm{m}^{3}$ of $\mathrm{GB}$ vapor for $10 \mathrm{~min}$. Electrocardiograms, electroencephalograms, and pupil size were monitored throughout exposure. Blood drawn before and throughout exposure was analyzed for blood gases, electrolytes, metabolites, acetylcholinesterase and BChE activities, and amount of GB bound to red blood cells and plasma. A novel finding was that saline-treated animals exposed to GB vapor did not develop any seizures, but manifested a variety of cardiac and whole blood toxic signs and rapidly died due to respiratory failure. Strikingly, pre-treatment with $7.5 \mathrm{mg} / \mathrm{kg}$ of $\mathrm{Hu} \mathrm{BChE}$ not only prevented lethality, but also avoided all cardiac toxic signs manifested in the non-treated cohort. Thus, Hu BChE alone can serve as an effective prophylactic countermeasure versus a lethal high-dose exposure to GB vapor.
\end{abstract}

Keywords: human serum butyrylcholinesterase, minipig, sarin vapor, high-dose exposure, cardiotoxicity 


\section{Introduction}

The physiological effects of organophosphorus (OP) pesticides and structurally-related chemical warfare nerve agents such as tabun (GA), sarin (GB), soman (GD) and VX, arise as a result of their ability to inhibit acetylcholinesterase (AChE) and the ensuing accumulation of acetylcholine [1, 2]. Exposure to nerve agents occurs by absorption through the skin (percutaneous) or lungs (inhalation) and impairs the activity of cholinergic synapses of the smooth and skeletal muscle, autonomic ganglia, and the central nervous system (CNS) [3, 4]. Due to their high volatility, "G-agents" pose a much greater inhalation hazard as compared to VX. The toxic effects of nerve agents depend on: (1) the concentration of agent, (2) the route of exposure, and (3) the duration of the exposure. Threshold symptoms that are commonly noted for vapor exposure are miosis, rhinorrhea, and airway constriction, generally appearing at a $C t$ (vapor concentration $X$ exposure time) of $2-3 \mathrm{mg} / \mathrm{min} / \mathrm{m}^{3}$ [5]. Low to moderate exposure of skin to liquid nerve agent causes localized sweating, nausea, vomiting, and a feeling of weakness [6]. Exposure to lethal amounts of nerve agent in liquid or vapor form gives rise to the multi-organ symptomology of acute nerve agent poisoning; miosis, stimulation of respiratory tract secretions, cardiovascular dysregulation, gastro-intestinal distress, muscle fasciculation and weakness, flaccid paralysis, cognitive and affective dysfunctions, ataxia, seizures, and respiratory collapse ultimately leading to death $[7,8]$.

Current military countermeasures against nerve agent threats combine carbamate pretreatment [9] followed by treatment with anti-cholinergics and oxime reactivators [10] in the event of nerve agent exposure. While effective in limiting the lethality of OP nerve agents, these measures exhibit a substantial adverse event profile and fail to address CNS exposure due to their inability to cross the blood-brain barrier. As a result, current treatment options do not 
prevent the development and progression of seizure activity, and the resultant neural and cardiac pathology $[9,11,12]$.

A number of novel approaches are currently being probed to augment the therapeutic options available to combat OP nerve agent toxicity. One such strategy is the use of either stoichiometric or catalytic bioscavengers, which would sequester and neutralize OP nerve agents prior to distribution into vital organ systems. Of the enzymes tested, serum-derived human butyrylcholinesterase (Hu BChE; EC 3.1.1.8) appears to be most appropriate for human use [13]. In this regard, numerous studies in rodent, guinea pig, and non-human primate preclinical models have now confirmed the favorable safety and pharmacokinetic profile of $\mathrm{Hu} \mathrm{BChE}$ [14-16], as well as its efficacy against the toxic signs and lethality of i.v., parenterally, or nose only administered OP nerve agents $[15,16-22]$. A dose of $200 \mathrm{mg}$ of $\mathrm{Hu} \mathrm{BChE}$ was proposed as a prophylactic treatment in humans that could protect from an exposure of up to $2 \mathrm{X} \mathrm{LD}_{50}$ of GD [23].

Inhalation is the most probable route of exposure to OPs on the battlefield or in public places and as mentioned above, the severity of symptoms is related to its concentration, which depend on the speed and direction of the wind and the volatility of the agent. In open air, lethal or immediately life-threatening results could be seen close to the site of agent release where the concentration is highest, while severe to moderate symptoms could be seen at some distance from that site. When released in a closed space, the volatile agent will disperse to fill the space; the smaller the space, the higher the concentration. We recently reported the toxic effects of exposing Göttingen minipigs to $4.1 \mathrm{mg} / \mathrm{m}^{3}$ of $\mathrm{GB}$ vapor for $60 \mathrm{~min}$ and the efficacy of $\mathrm{Hu} \mathrm{BChE}$ as a prophylactic measure against this exposure [24]. In this study, all untreated animals died due to respiratory failure and/or seizures. However, pretreatment with $7.5 \mathrm{mg} / \mathrm{kg}$ of $\mathrm{Hu} \mathrm{BChE}$ 
alone was not only effective in increasing survivability, but also prevented cardiac abnormalities and neural toxicity observed in untreated minipigs.

In the present study, we investigated the toxic effects of an approximately 3-fold higher dose of GB vapor in Göttingen minipigs. We also investigated the ability of $\mathrm{Hu} \mathrm{BChE}$ pretreatment (administered by i.m. injection, $24 \mathrm{~h}$ prior to exposure) to protect against a rapid whole-body exposure of $11.4 \mathrm{mg} / \mathrm{m}^{3}$ of $\mathrm{GB}$ vapor for $10 \mathrm{~min}$, a regimen designed to more closely model a real-world nerve agent exposure. Electrocardiogram (ECG), electroencephalograms (EEG), and pupil size, were monitored throughout exposure. Blood drawn before and throughout exposure was analyzed for blood gases, electrolytes, metabolites, AChE and BChE activities, and amount of GB bound to plasma and red blood cells (RBCs). Results showed that saline-treated animals exposed to a high-dose of GB vapor manifested a variety of cardiac and whole blood toxic signs, and died due to respiratory failure. Furthermore,

unlike animals that were exposed to $4.1 \mathrm{mg} / \mathrm{m}^{3}$ of GB vapor for $60 \mathrm{~min}$, these minipigs did not develop any generalized seizures. Pre-treatment of minipigs with $7.5 \mathrm{mg} / \mathrm{kg}$ of $\mathrm{Hu} \mathrm{BChE}$ not only increased survival, but also prevented all cardiac toxic signs manifested in the un-treated cohort.

\section{Materials and Methods}

\subsection{Materials}

All reagent grade chemicals including acetylthiocholine (ATC), butyrylthiocholine (BTC), 5,5-dithiobisnitrobenzoic acid (DTNB), and ethopropazine were purchased from Sigma Chemical Co. (St. Louis, MO). Hu BChE was isolated from Cohn fraction IV-4 paste [25] and stored in lyophilized form at $-20^{\circ} \mathrm{C}$. 


\subsection{Procedure for Whole-Body Inhalation Exposure to Sarin Vapor}

All animal studies were conducted in compliance with the Animal Welfare Act and other federal statutes and regulations stated in the Guide for the Care and Use of Laboratory Animals (NRC Publication, 1996 edition). All procedures with animals received prior approval from the Edgewood Chemical and Biological Center (ECBC) Institutional Animal Care and Use Committee and performed in facilities fully accredited by the Association for Assessment and Accreditation of Laboratory Animal Care, International. Sexually mature male Göttingen minipigs (Sus scrofa) obtained from Marshall Farms USA (North Rose, NY) were used for these studies. Indwelling catheters were implanted into the external jugular veins of the minipigs as described [26]. The minipigs were allowed to recover for at least three days before they were used for any exposures. During that time, the catheter was flushed with heparinized saline as needed.

$O$-Isopropyl methylphosphonofluoridate (sarin or GB) used in this study was obtained from the Chemical Transfer Facility, ECBC, APG, MD. Chemical agent standard analytical reagent material (CASARM)-grade GB (lot GB-U-4065-CTF-N) was verified as $97.3 \pm 0.74$ wt $\%$ pure by quantitative ${ }^{31} \mathrm{P}$ NMR spectroscopy and stored in sealed ampoules under nitrogen.

Whole-body exposures to GB vapor were conducted by the Operational Toxicology

Team, Research and Technology Directorate at ECBC, APG, MD using a 1000-1 dynamic airflow chamber, as described [26]. Briefly, each pig was placed and maintained in a canvas sling (Lomir Biomedical, Inc., Malone, NY, or Canvas and Awning supplies, White Marsh, MD). The jugular line exteriorized from the exposure chamber, was used for the collection of blood samples throughout the exposure period. Dermal electrodes were attached and exteriorized from 
the exposure chamber for ECG and EEG recordings using a Ceegraph Netlink headbox with the Sleepscan computer system (Bio-Logic Systems Corp, Mundelein, IL). Infrared images of the pupil were taken through the Plexiglas.

\subsection{Efficacy of Hu BChE in Minipigs}

Minipigs were subjected to one of the following treatments: (1) saline injection followed by air exposure $(n=4)$; (2) saline injection followed by GB vapor exposure $(n=3)$; and (3) pretreatment with $7.5 \mathrm{mg} / \mathrm{kg}$ of $\mathrm{Hu} \mathrm{BChE}$ followed by GB vapor exposure $(\mathrm{n}=4) . \mathrm{Hu} \mathrm{BChE}$ or saline was administered by i.m. injection, $24 \mathrm{~h}$ prior to whole-body exposure to GB vapor. Pharmacokinetic studies conducted previously showed that $\mathrm{Hu}$ BChE delivered into minipigs by i.m. injection attained maximal blood concentration at $24 \mathrm{~h}$. The concentration of GB vapor used was $11.4 \mathrm{mg} / \mathrm{m}^{3}$ for $10 \mathrm{~min}$ yielding an exposure of $114 \mathrm{mg} \cdot \mathrm{min} / \mathrm{m}^{3}$, which is equivalent to $\mathrm{LCt}_{99}$. The experimentally generated value of $\operatorname{LC}_{50}\left(7.25 \mathrm{mg} / \mathrm{m}^{3}\right)$ for the $10 \mathrm{~min}$ exposure was extrapolated to calculate the value of $\operatorname{LC}_{99}\left(11.4 \mathrm{mg} / \mathrm{m}^{3}\right)$ using the probit slope as described [26]. EEG, ECG, and pupil size, were monitored throughout exposure. Blood drawn from a surgically implanted jugular catheter before and at 2, 4, 6, 8, 10, 20, 30, and 60 min following exposure to GB vapor was analyzed for blood gases, electrolytes, metabolites, AChE and BChE activities, and the amount of GB present in plasma and RBCs.

\subsection{Blood Assays}

Following collection, blood samples were analyzed immediately for $\mathrm{pH}$, partial pressure carbon dioxide $\left(\mathrm{pCO}_{2}\right)$, partial pressure oxygen $\left(\mathrm{pO}_{2}\right), \mathrm{Na}^{+}, \mathrm{K}^{+}$, hematocrit $(\mathrm{HCT})$, and glucose using an i-STAT Portable Clinical Analyzer (i-STAT Corp., Princeton, NJ) and EG8+ cartridges. 
Values for bicarbonate $\left(\mathrm{HCO}_{3}{ }^{-}\right)$, hemoglobin $(\mathrm{Hb}), \mathrm{TCO}_{2}$ (total $\left.\mathrm{CO}_{2}\right)$, and $\mathrm{sO}_{2}$ (oxygen saturation) were also obtained.

AChE and BChE activities in whole blood were measured using a modified Ellman assay $[27,28]$ as described previously [24]. One unit (U) of enzyme activity is defined as the amount that hydrolyzes one $\mu$ mol of substrate in one min.

Blood was separated into erythrocyte and plasma fractions by centrifugation and the amount of GB bound to each fraction was determined by measuring regenerated GB using gas chromatography/mass spectrometry (GC/MS) as described [24, 29].

\subsection{Analysis of ECG and EEG data}

Analysis of ECG data was performed using ECG-AUTO software (Emka Technologies, Falls Church, VA) as described [24]. Briefly, waveform libraries were established for each subject and analyzed in 2 min contiguous time blocks, with a minimum of 20 valid beats required to qualify the data block for statistical analysis. For each block, RR, PR, and QT intervals were quantified. Heart rate (HR) was calculated from the RR interval, and QT intervals were corrected for HR using a linear regression approach as previously described [30]. The final $10 \mathrm{~min}$ of the chamber acclimation period were taken as the pre-exposure baseline for each animal. The following $60 \mathrm{~min}$, which included the $10 \mathrm{~min}$ exposure period, were then analyzed. The ECG for all animals surviving GB exposure was re-evaluated 1 week later.

Following acclimation of pigs to the inhalation chamber, EEG for a pre-exposure baseline of at least $20 \mathrm{~min}$, and a $60 \mathrm{~min}$ period following introduction of agent were recorded. EEG data were analyzed using the Sleepscan II and Insight software modules of the Bio-logic system as described previously [24]. The fronto-central (Fz-Cz) montage was initially screened 
by visual inspection to disqualify regions of the tracing exhibiting electromuscular contamination or other artifact. Low- $(<0.5 \mathrm{~Hz})$ and high-band $(>35 \mathrm{~Hz})$ filters, as well as a $60 \mathrm{~Hz}$ notch filter were then applied to the tracings. Tracings were analyzed in two ways: (1) the entire $60 \mathrm{~min}$ monitoring session was fragmented into contiguous time segments, and a 60 sec data block was subject to spectral analysis after applying Fast Fourier Transformation (FFT), and (2) the $10 \mathrm{~min}$ GB exposure period was fragmented into 2 min segments with $30 \mathrm{sec}$ data blocks evaluated by spectral analysis. EEG power in each of the classical frequency bands was determined by integrating the frequency spectrum using the following limits: delta $=0.5-4.5 \mathrm{~Hz}$, theta $=4.5-$ $8.5 \mathrm{~Hz}$, alpha $=8.5-13.5 \mathrm{~Hz}$, beta $1=13.5-21.5 \mathrm{~Hz}$, and beta $2=21.5-35.5 \mathrm{~Hz}$.

\subsection{Statistical Analysis}

\subsubsection{ECG:}

Correlation Analysis for $R R-Q T$ Intervals: QT interval dependence as a function of HR (RR interval) was examined using bivariate regression analysis as previously described (Sagie $e t$ al., 1992). The resulting regression constants were used to correct the QT interval (referred to herein as QTlc) using a literature value of $95 \mathrm{bpm}$ for $\mathrm{HR}$.

Longitudinal Analysis of Cardiac Parameters: Individual variance with respect to the onset of GB cardiac toxic signs precluded a conventional repeated measures analysis. To address this issue, dataset were synchronized to the onset of toxic signs as described [24] to assess whether Hu BChE pretreatment prevented or attenuated GB-induced cardiac abnormalities. Once aligned in this manner, the data were analyzed by means of a repeated measures analysis of variance (RMA). As the datasets were found to deviate from compound symmetry, univariate F-ratios 
were tested against Greenhouse-Geisser corrected degrees of freedom. The resulting approximate F-ratio is reported, with alpha $=0.05$. Post-hoc comparison of $\mathrm{Hu} \mathrm{BChE}$ treatment profile with that of air-exposed control pigs was performed using the Dunnett procedure to constrain the multiple contrast error rate to an acceptable level.

\subsubsection{EEG Spectra:}

Longitudinal analysis of EEG spectra was performed using RMA as described above. Hu BChE treatment, classical frequency bands and their interaction were considered betweensubjects effects, while time from agent onset and the interaction of time with each betweensubjects factor were considered within-subject effects. Post-hoc comparisons of spectral profile from treated, un-treated, and air-exposed control pigs were performed using the Dunnett procedure to constrain the multiple contrast error rate.

\section{Results}

\subsection{Circulating cholinesterase activity following exposure to GB vapor}

Minipigs were pretreated with saline or $\mathrm{Hu} \mathrm{BChE}$ and challenged with air or an acute dose of GB vapor $\left(11.4 \mathrm{mg} / \mathrm{m}^{3}\right.$ for $\left.10 \mathrm{~min}\right) 24 \mathrm{~h}$ later. The average baseline BChE activity in the blood of saline-treated animals was $0.16 \pm 0.03 \mathrm{U} / \mathrm{ml}$, which reached $39.7 \pm 10.5 \mathrm{U} / \mathrm{ml}$ at $24 \mathrm{~h}$ following the administration of $7.5 \mathrm{mg} / \mathrm{kg}$ of $\mathrm{Hu} \mathrm{BChE}$ by i.m. injection. The administration of this dose of $\mathrm{Hu}$ BChE did not affect circulating AChE activity $(2.21 \pm 0.3 \mathrm{U} / \mathrm{ml})$. When saline-

treated animals were exposed to an acute dose of GB vapor $\left(11.4 \mathrm{mg} / \mathrm{m}^{3}\right), \sim 90 \%$ of circulating AChE activity was inhibited at $10 \mathrm{~min}$ into exposure (Figure $1 \mathrm{~B}$ ). At this time, GB vapor in the chamber was replaced by air and the animals were kept in the chamber for another 50 min and 
blood from surviving animals was sampled for up to $2 \mathrm{~h}$ from the onset of GB vapor exposure. Circulating $\mathrm{AChE}$ and $\mathrm{BChE}$ activities at $60 \mathrm{~min}$ were $7 \%$ and $15 \%$ of peak activities, respectively. All untreated control animals died at 10-23 min into GB vapor exposure, while all four animals pretreated with $7.5 \mathrm{mg} / \mathrm{kg}$ of $\mathrm{Hu} \mathrm{BChE}$ survived exposure to GB vapor. As shown in Figure 1, exogenously administered $\mathrm{Hu}$ BChE delayed the inhibition of circulating AChE activity (Figure 1B) by neutralizing GB in circulation (Figure 1A) and prevented toxicity due to GB vapor. This was also substantiated by the results of a fluoride ion-based regeneration assay that measured the amount of GB bound to RBCs and plasma at 10 min into exposure. Since minipig blood contains a very low level of circulating BChE activity, most of the GB was bound to RBCs and essentially no GB was recovered from the plasma of untreated animals exposed to GB vapor (Figure 2). On the other hand, most of the GB was bound to plasma in minipigs that were pretreated with $7.5 \mathrm{mg} / \mathrm{kg}$ of $\mathrm{Hu} \mathrm{BChE}$ (Figure 2). The amount of GB detected in RBCs from all $\mathrm{Hu} \mathrm{BChE}$ pretreated pigs was significantly less than that from control animals.

\subsection{Effect of $\mathrm{Hu}$ BChE pretreatment on GB vapor-induced changes in blood chemistry} parameters

Changes in blood chemistry parameters were monitored in minipigs that were exposed to GB vapor following pretreatment with saline (untreated) or $7.5 \mathrm{mg} / \mathrm{kg}$ of $\mathrm{Hu} \mathrm{BChE}$. Values prior to initiation of exposure and at $60 \mathrm{~min}$ post-GB vapor $(10 \mathrm{~min} \mathrm{~GB}+50 \mathrm{~min}$ air) exposure are shown in Table 1. Minipigs that did not receive Hu BChE pretreatment died at 10-23 min into GB vapor exposure, which precluded the collection of blood samples after 20 min. Changes in blood chemistry parameters are consistent with an acute respiratory failure characterized by hypoxemia, hypercapnia, acute respiratory acidosis, and low blood $\mathrm{sO}_{2}$. In untreated animals, 
blood $\mathrm{pO}_{2}$ levels started to decline within 4 min into $\mathrm{GB}$ vapor exposure, accompanied by a sharp increase in blood $\mathrm{pCO}_{2}$, a decrease in blood $\mathrm{HCO}_{3}{ }^{-}$and $\mathrm{pH}$. Changes in blood $\mathrm{pCO}_{2}$ and $\mathrm{HCO}_{3}{ }^{-}$indicated both metabolic acidosis and respiratory acidosis due to respiratory failure. Although not as dramatic as those in the previous study, increases in serum glucose levels and $\mathrm{Hb}$ were also observed. However, the blood chemistry parameters of GB-exposed pigs pretreated with $7.5 \mathrm{mg} / \mathrm{kg} \mathrm{Hu} \mathrm{BChE}$ were essentially similar to those of air-exposed pigs, suggesting that pretreatment with this dose of enzyme prevented respiratory failure.

\subsection{Baseline ECG and EEG parameters in control minipigs}

Four air-exposed control subjects used in our previous study [24] were re-analyzed in parallel with the baseline values for minipigs used in the present study to establish within-study reference values for cardiac HR, PR, and QTlc intervals $(n=13)$. These parameters (data not shown) were generally in-line with previously reported data, although HR and ventricular repolarization appears to be more rapid in our animals. RR and QT intervals were found to be significantly correlated $\left[\mathrm{r}^{2}=0.65, \mathrm{p}<0.05\right]$. The use of the regression coefficients successfully eliminated the correlation of these two parameters $\left[\mathrm{r}^{2}=0.0005\right]$. PR intervals for air-exposed pigs were similar to reference values.

Consistent with observations made in our previous study [24], a typical example of an EEG power spectrum obtained from an air-exposed pig (not shown) demonstrated low amplitude (peak $<300 \mu \mathrm{V}$ ), long wavelength energy predominantly within the delta and theta frequency bands. When plotted as a function of time, EEG power spectra of control pigs were stable throughout the period of air exposure. 


\subsection{Effect of Hu BChE pretreatment on GB vapor-induced cardiac abnormalities}

As shown in Figure 3, whole-body inhalation exposure to GB vapor $\left(11.4 \mathrm{mg} / \mathrm{m}^{3}\right.$ for 10 min) resulted in the rapid development of a variety of cardiac abnormalities in the minipig. Tachycardia served as the initial indication of cardiac abnormality and began within several min of GB exposure. HR accelerated throughout the $10 \mathrm{~min}$ GB exposure period, reaching nearly 200 bpm. PR and uncorrected QT intervals were largely unchanged during this time. A second phase of cardiac abnormality was marked by the development of a profound bradycardia occurring between 10 and 20 min following exposure. Interestingly, this period was associated with a slight shortening, rather than lengthening, of the PR interval suggesting the development of cardiac arrhythmia. In a final phase of toxicity, HR dramatically slowed concomitant with a marked lengthening of the PR interval. QT intervals remained stable until the animals expired. A regular, but progressively slowing junctional or ventricular rhythm was observed immediately prior to complete cardiac arrest. Neither QT prolongation nor end-stage tachycardia were features of acute GB intoxication in the minipig.

All animals ( $\mathrm{n}=4$ ) pre-treated with $7.5 \mathrm{mg} / \mathrm{kg}$ of $\mathrm{Hu} \mathrm{BChE}$ survived the acute exposure to GB vapor. As noted previously, pretreatment with $\mathrm{Hu} \mathrm{BChE}$ tended to result in a reduced baseline HR (Saxena et al., 2011a). HR, PR, and QT intervals remained unchanged from baseline levels throughout GB vapor exposure and subsequent 50 min post-exposure monitoring period (Figure 3). Thus, pretreatment of minipigs with $\mathrm{Hu} \mathrm{BChE} 24 \mathrm{~h}$ prior to an acute exposure to GB vapor appeared to completely prevent cardiac abnormalities. To confirm protection against chronic cardiac abnormalities resulting from GB vapor exposure, ECGs from $\mathrm{Hu}$ BChEpretreated pigs were re-evaluated 1 week following GB exposure. As shown in Figure 4, HR, PR and QT1c intervals were unchanged from pre-GB exposure baselines. 


\subsection{Effect of Hu BChE pretreatment on GB vapor-induced neurotoxicity}

As indicated above, all saline-treated minipigs rapidly succumbed to GB vapor, while $\mathrm{Hu}$ BChE-pretreated animals survived the acute exposure to GB vapor. The EEG spectra for salineand Hu BChE-pretreated groups are shown in Figure 5. As shown in Figure 5B, saline-pretreated minipigs exposed to GB vapor via an acute dosing approach $\left(11.4 \mathrm{mg} / \mathrm{m}^{3}\right.$ for $\left.10 \mathrm{~min}\right)$ did not develop generalized tonic-clonic seizures observed in the previous study [24]. With the onset of GB vapor exposure, a slight increase in overall power over baseline level was observed in both saline- and $\mathrm{Hu}$ BChE-pretreated animals (Figures 5B and 5C, respectively). Peak power, however, remained predominantly in the delta frequency band, below $400 \mu \mathrm{V}$ (Figure 5C), and on par with the spectral distribution of air-exposed pigs (Figure 5A). EEG spectra from $\mathrm{Hu}$ BChE-pretreated pigs exposed to GB vapor were re-evaluated 1 week following GB exposure. As shown in Figure 5D, EEG spectra from Hu BChE-pretreated pigs exposed to GB vapor reevaluated 1 week following GB exposure appear relatively normal.

\subsection{Effect of Hu BChE pretreatment on GB vapor-induced miosis}

Both saline- and Hu BChE-pretreated pigs developed miosis within 10 min of exposure, with the earliest onset recorded at $7.5 \mathrm{~min}$. Miosis is a direct effect of OP nerve agent exposure on the eyes [31] and was not affected by pretreatment with $\mathrm{Hu} \mathrm{BChE}$. However, miosis resolved within a week.

\section{Discussion}

Several alternatives to the current carbamate pretreatment regimen are being considered as prophylactic nerve agent countermeasures, including the development of high affinity native 
and recombinant cholinesterases (ChEs) and OP hydrolases [32]. From a safety standpoint, Hu BChE appears to be the most suitable for immediate development. The use of native BChE not only avoids immunogenicity in man that could limit in vivo availability, but also reduces the likelihood of significant adverse events. Since G-agents exist as volatile liquids at normal ambient temperatures, inhalation is the most likely route of exposure to these agents. Thus, efforts were initiated to evaluate the efficacy of $\mathrm{Hu}$ BChE against whole-body inhalation exposure to a G-agent, GB. Two studies were conducted using different lethal doses for GB vapor exposure. In the first study, minipigs exposed to $4.1 \mathrm{mg} / \mathrm{m}^{3}$ of GB vapor for $60 \mathrm{~min}$ died at $45.5 \pm 7.9 \mathrm{~min}$ [24]. In the present study, minipigs subjected to an acute challenge of 11.4 $\mathrm{mg} / \mathrm{m}^{3}$ of $\mathrm{GB}$ vapor for $10 \mathrm{~min}$ died at $17.7 \pm 6.8 \mathrm{~min}$. These studies not only highlight differences in dose-dependent toxicity of a lethal dose of inhaled GB vapor, but also demonstrate the efficacy of $\mathrm{Hu}$ BChE pretreatment in alleviating toxicity in a large animal model.

All untreated minipigs exposed to $4.1 \mathrm{mg} / \mathrm{m}^{3}$ of $\mathrm{GB}$ vapor for $60 \mathrm{~min}$ developed severe respiratory stress characterized by hypoxemia, hypercapnia, and acute respiratory acidosis and ultimately died due to respiratory failure [24]. Minipigs challenged with $11.4 \mathrm{mg} / \mathrm{m}^{3}$ of GB vapor for $10 \mathrm{~min}$ also displayed signs of severe respiratory stress and died due to respiratory failure. The effects on the respiratory system are mediated by the binding of accumulated acetylcholine to both muscarinic and nicotinic receptors [31]. Muscarinic effects including rhinorrhea and bronchial secretion, bronchospasm, and laryngeal spasm, can cause airway obstruction while nicotinic effects can cause the paralysis of respiratory muscles. A weakness of the muscles in tongue and pharynx can also aggravate upper airway obstruction. In addition, OPs can act directly at cholinergic synapses in the brain stem that control respiration, leading to 
cessation of breathing $[4,8]$. In humans, the effect on the central respiratory drive is the primary reason for respiratory failure and the peripheral neuromuscular block is secondary $[7,8]$.

In both studies, untreated animals exposed to GB vapor displayed a variety of cardiovascular effects, which are the net result of excitatory and inhibitory actions of accumulated acetylcholine at ganglionic, medullary, vasomotor, and cardiac centers [34]. These effects are further complicated by hypoxia, local release of catecholamines, and disturbances of ion transport. Ludomirsky et al., described three phases of cardiotoxicity, which are responsible for producing various changes in ECG [35]: (1) an initial sympathetic phase produces sinus tachycardia; (2) a second prolonged parasympathetic phase manifests as ST-T changes and A-V conduction disturbances; and (3) a third phase of QT prolongation, ventricular tachycardia and fibrillation immediately prior to cardiac death. In the present study, cardiac abnormalities included sinus tachycardia followed by profound sinus bradycardia and the development of cardiac arrhythmia. In a final phase of toxicity, HR dramatically slowed concomitant with a marked lengthening of the PR interval. Neither QT prolongation nor end-stage tachycardia, were features of acute GB intoxication in the minipig. QTc prolongation was previously observed in rats that survived exposure to GD or GB by a bolus injection or to GB vapor [36-38]. This effect was observed in rats exhibiting critical toxicity signs during or immediately following exposure and was limited to the first two weeks following intoxication [38]. QT prolongation during the first 2-3 weeks was also reported in humans accidently exposed to OP pesticides [35, 39]. However, QT prolongation did not correlate with cardiac and respiratory failure leading to death $[38,39]$. The absence of QT prolongation in the minipig may reflect a difference in species or a difference in exposure paradigm. 
In this context, it should also be noted that both hypoxemia and hyperkalemia observed in untreated animals exposed to GB vapor, could also have contributed to the cardiotoxic effects. Hyperglycemia and hyperkalemia were also reported in domestic pigs that were exposed to GD percutaneously [40], suggesting a similarity in the mode of action of G-agents. $\mathrm{K}^{+}$is predominantly an intracellular cation and plays an important role in maintaining electrical potential across the cell membrane as well as in depolarization and repolarization of myocytes. Thus, alterations in serum $\mathrm{K}^{+}$levels can have dramatic effects on cardiac cell conduction and many studies have demonstrated a good correlation between hyperkalemia and changes in ECG [41]. In addition, the magnitude of abnormalities in ECG is proportional to serum $\mathrm{K}^{+}$levels. The earliest ECG abnormality reported in patients with serum $\mathrm{K}^{+}$levels as low as $5.5 \mathrm{mM}$ is $\mathrm{T}$ wave tenting resulting from the acceleration of the terminal phase of repolarization. With higher levels of $\mathrm{K}^{+}$exceeding $6.5 \mathrm{mM}$, cardiac conduction between myocytes is suppressed, resulting in $\mathrm{P}$ wave flattening and PR interval prolongation. When serum $\mathrm{K}^{+}$levels exceed two times the normal value, there is suppression of sinoatrial and $\mathrm{A}-\mathrm{V}$ conduction, resulting in sinoatrial and A-V blocks.

In the previous study, we demonstrated that all animals pretreated with $7.5 \mathrm{mg} / \mathrm{kg}$ of $\mathrm{Hu}$ BChE survived the $60 \mathrm{~min}$ exposure to $4.1 \mathrm{mg} / \mathrm{m}^{3}$ of GB vapor [24]. Thus, the efficacy of $\mathrm{Hu}$ $\mathrm{BChE}$ in this study was evaluated using the same dose of enzyme. Although the rate of GB vapor exposure was almost 3-fold higher than in the previous study, pretreatment with $\mathrm{Hu} \mathrm{BChE}$ was highly effective not only in preventing lethality, but also in avoiding all cardiac and whole blood toxic signs manifested in untreated minipigs. The protection afforded by $\mathrm{Hu} \mathrm{BChE}$ was due to the rapid binding and neutralization of GB in circulation, as demonstrated by: (1) assays of circulating $\mathrm{AChE}$ and $\mathrm{BChE}$ activities, which showed a delay in inhibition of AChE activity in 
Hu BChE-pretreated animals; (2) results of fluoride ion-based regeneration assay, which showed that the amount of GB detected in RBCs from $\mathrm{Hu}$ BChE-pretreated minipigs was significantly less than that from control animals; and (3) analysis of blood chemistry parameters, which showed that parameters for GB-exposed pigs pretreated with $\mathrm{Hu} B C h E$ were similar to those of air-exposed control pigs.

A novel finding of the present study was that minipigs exposed to $11.4 \mathrm{mg} / \mathrm{m}^{3}$ of GB vapor for $10 \mathrm{~min}$ did not develop generalized tonic-clonic seizures as indicated by a relatively normal EEG spectrum. All saline-treated minipigs rapidly succumbed to GB vapor, while $\mathrm{Hu}$ BChE- pretreated animals survived the acute exposure to GB vapor, but EEG spectra remained relatively normal for both groups. A slight increase in overall power over baseline level observed in both $\mathrm{Hu}$ BChE- and saline-treated animals with the onset of GB vapor exposure (Figure 5), is perhaps related to stimulus presentation, e.g. airflow changes in the inhalation chamber and does not reflect a nerve agent-specific event. In contrast, minipigs subjected to a 60 min whole-body inhalation exposure to $4.1 \mathrm{mg} / \mathrm{m}^{3}$ of GB vapor exhibited a marked increase in total power (peak amplitude of $600 \mu \mathrm{V}$ ) with the onset of generalized seizures 30-40 min into exposure to GB vapor and FFT analysis revealed a redistribution of the power spectra into high frequency bands [24].

In light of the rapid development of cardiac toxic signs and the quick demise of the untreated animals exposed to $11.4 \mathrm{mg} / \mathrm{m}^{3}$ of GB vapor, it is clear that the lack of EEG findings is not related to an issue with agent exposure. Moreover, real-time monitoring of the chamber atmosphere confirmed a $>11 \mathrm{mg} / \mathrm{m}^{3}$ steady state concentration of GB, approximately three times that which produced seizures in the previous study. In addition, monitoring of blood chemistry parameters revealed a profound hypercapnia and respiratory acidosis which presaged complete 
respiratory collapse. All of these features were completely absent in pigs pretreated with $\mathrm{Hu}$ BChE. Thus, it seems most likely that the untreated animals in the present study simply succumbed to intoxication prior to the onset of seizure activity. Because of the absence of seizures in the untreated group, the present study does not directly address shielding of CNS effects by $\mathrm{Hu} \mathrm{BChE}$ under these exposure conditions. It is, however, encouraging to note that $\mathrm{Hu}$ BChE-pretreated minipigs failed to develop abnormal EEG signs at any point during the postexposure period or in a 1 week follow-up examination. Taken together, these results demonstrate that a dose of $7.5 \mathrm{mg} / \mathrm{kg}$ of $\mathrm{Hu} \mathrm{BChE}$ alone was not only effective in increasing survivability, but also prevented cardiac toxicity in minipigs subjected to an acute lethal high dose challenge with GB vapor.

\section{Conclusions}

The present report investigated the toxicity of a high dose of GB vapor in the Göttingen minipig and whether pretreatment with $\mathrm{Hu}$ BChE could prevent toxicity and lethality in exposed animals. Untreated minipigs challenged with $11.4 \mathrm{mg} / \mathrm{m}^{3}$ of GB vapor for $10 \mathrm{~min}$, exhibited a variety of signs indicating cardiac toxicity, which included significant and progressive heart rate anomalies, arrhythmia, and, ultimately, cardiac arrest. In addition, monitoring of blood chemistry revealed a profound hypercapnia and respiratory acidosis which presaged complete respiratory collapse. However, untreated pigs failed to develop epileptiform activity, suggesting that the animals succumbed to GB intoxication - most likely due to respiratory failure prior to the manifestation of EEG toxic signs. These symptoms of cardiac and whole-blood toxicity were completely absent in minipigs that were pretreated with $7.5 \mathrm{mg} / \mathrm{kg}$ of $\mathrm{Hu}$ BChE. Taken together, 
these results demonstrate that $\mathrm{Hu} \mathrm{BChE}$ pretreatment alone was effective against a lethal high dose exposure to GB vapor.

\section{Acknowledgements}

The opinions or assertions contained herein are the private views of the authors and are not to be construed as official or as reflecting the views of the Army or the Department of Defense. The authors wish to thank Ms. Amy Michels for her assistance in preparation of the manuscript. This research was supported by funding from the Defense Threat Reduction Agency.

\section{References}

[1] Koelle GB. Cholinesterases and anticholinesterases. In: Ekhler O, Farah A, editors. Handbuch der Experimentallen Pharmakologie. Berlin: Springer-Verlag, 1963.

[2] Taylor P. Anticholinesterase Agents. In: Gilman AG, Rall TW, Nies AS, Taylor P, editors. The Pharmacological Basis of Therapeutics. New York: Macmillan, 1990. p. 131.

[3] Heffron PF, Hobbiger F. Relationship between inhibition of acetylcholinesterase and response of the rat phrenic nerve-diaphragm preparation to indirect stimulation at higher frequencies. Br J Pharmacol 1979;66:323-9.

[4] Stewart WC, Anderson EA. Effect of a cholinesterase inhibitor when injected into the medulla of the rabbit. J Pharmacol Exp Ther 1968;162:309-18.

[5] Sidell FR. Chemical considerations in nerve agent intoxication. In, Chemical warfare Agents, ed. S. Somani, New York, Academic Press, 1992, pp.155-194.

[6] Medical Management of Chemical Casualties Handbook, Third Edition, US Army Medical Research Institute of Chemical Defense, Chemical Casualty Care Office. Aberdeen Proving Ground, MD, 2000. 
[7] Stewart WC. The effects of sarin and atropine on the respiratory center and neuromuscular junctions of the rat. Can J Biochem Physiol 1959;37:651-60.

[8] de Candole CA, Douglas WW, Evans CL, Holmes R, Spencer KE, Torrance RW, et al. The failure of respiration in death by anticholinesterase poisoning. Br J Pharmacol Chemother 1953;8:466-75.

[9] Dirnhuber P, French MC, Green DM, Leadbeater L, Stratton JA. The protection of primates against soman poisoning by pretreatment with pyridostigmine. J Pharm Pharmacol 1979;31:295-9.

[10] Gray AP. Design and structure-activity relationships of antidotes to organophosphorus anticholinesterase agents. Drug Metab Rev 1984;15:557-89.

[11] McLeod CGJ. Pathology of nerve agents: Perspectives on medical management. Fundam Appl Toxicol 1985;5:S10-S6.

[12] Dunn MA, Sidell FR. Progress in medical defense against nerve agents. JAMA 1989;262:649-52.

[13] Ashani Y. Prospective of human butyrylcholinesterase as a detoxifying antidote and potential regulator of controlled-release drugs. Drug Dev Res 2000;50:298-308.

[14] Saxena A, Sun W, Luo C, Doctor BP. Human serum butyrylcholinesterase: in vitro and in vivo stability, pharmacokinetics, and safety in mice. Chemico-biological interactions 2005;157-158:199-203.

[15] Lenz DE, Maxwell DM, Koplovitz I, Clark CR, Capacio BR, Cerasoli DM, et al. Protection against soman or VX poisoning by human butyrylcholinesterase in guinea pigs and cynomolgus monkeys. Chemico-biological interactions 2005;157-158:205-10.

[16] Clark MG, Sun W, Myers TM, Bansal R, Doctor BP, Saxena A. Effects of physostigmine and human butyrylcholinesterase on acoustic startle reflex and prepulse inhibition in C57BL/6J mice. Pharmacol Biochem Behav 2005;81:497-505.

[17] Ashani Y, Shapira S, Levy D, Wolfe AD, Doctor BP, Raveh L. Butyrylcholinesterase and acetylcholinesterase prophylaxis against soman poisoning in mice. Biochem Pharmacol 1991;41:37-41.

[18] Raveh L, Grunwald J, Marcus D, Papier Y, Cohen E, Ashani Y. Human butyrylcholinesterase as a general prophylactic antidote for nerve agent toxicity. In vitro and in vivo quantitative characterization. Biochem Pharmacol 1993;45:2465-74. 
[19] Brandeis R, Raveh L, Grunwald J, Cohen E, Ashani Y. Prevention of soman-induced cognitive deficits by pretreatment with human butyrylcholinesterase in rats. Pharmacol Biochem Behav 1993;46:889-96.

[20] Raveh L, Grauer E, Grunwald J, Cohen E, Ashani Y. The stoichiometry of protection against soman and VX toxicity in monkeys pretreated with human butyrylcholinesterase. Toxicol Appl Pharmacol 1997;145:43-53.

[21] Allon N, Raveh L, Gilat E, Cohen E, Grunwald J, Ashani Y. Prophylaxis against soman inhalation toxicity in guinea pigs by pretreatment alone with human serum butyrylcholinesterase. Toxicol Sci 1998;43:121-8.

[22] Saxena A, Sun W, Fedorko JM, Koplovitz I, Doctor BP. Prophylaxis with human serum butyrylcholinesterase protects guinea pigs exposed to multiple lethal doses of soman or VX. Biochem Pharmacol 2011;81:164-9.

[23] Ashani Y, Pistinner S. Estimation of the upper limit of human butyrylcholinesterase dose required for protection against organophosphates toxicity: a mathematically based toxicokinetic model. Toxicol Sci 2004;77:358-67.

[24] Saxena A, Sun W, Dabisch PA, Hulet SW, Hastings NB, Jakubowski EM, et al. Pretreatment with human serum butyrylcholinesterase alone prevents cardiac abnormalities, seizures, and death in Gottingen minipigs exposed to sarin vapor. Biochem Pharmacol 2011;82:1984-93.

[25] Saxena A, Tipparaju P, Luo C, Doctor BP. Pilot-scale production of human serum butyrylcholinesterase suitable for use as a bioscavenger against nerve agent toxicity. Process Biochemistry 2010;45:1313-8.

[26] Hulet SW, Sommerville DR, Miller DB, Scotto JA, Muse WT, Burnett DC. Comparison of sarin and cyclosarin toxicity by subcutaneous, intravenous and inhalation exposure in Gottingen minipigs. Inhal Toxicol. 2014;26:175-84.

[27] Ellman GL, Courtney KD, Andres V, Jr., Feather-Stone RM. A new and rapid colorimetric determination of acetylcholinesterase activity. Biochem Pharmacol 1961;7:88-95.

[28] Worek F, Mast U, Kiderlen D, Diepold C, Eyer P. Improved determination of acetylcholinesterase activity in human whole blood. Clin Chim Acta 1999;288:73-90.

[29] Jakubowski EM, McGuire JM, Evans RA, Edwards JL, Hulet SW, Benton BJ, et al. Quantitation of fluoride ion released sarin in red blood cell samples by gas 
chromatography-chemical ionization mass spectrometry using isotope dilution and largevolume injection. J Anal Toxicol 2004;28:357-63.

[30] Sagie A, Larson MG, Goldberg RJ, Bengtson JR, Levy D. An improved method for adjusting the QT interval for heart rate (the Framingham Heart Study). Am J Cardiol 1992;70:797-801.

[31] Sim VM. Effect on pupil size of exposure to GB vapor. Directorate of Chemical Defense Research and Development, 1956.

[32] Saxena A, Luo C, Chilukuri N, Maxwell DM, Doctor BP. Novel approaches to medical protection against chemical warfare nerve agents. In: Romano JAJ, Lukey BJ, Salem H, editors. Chemical Warfare Agents Chemistry, Pharmacology, and Therapeutics. Boca Raton, FL: CRC Press, 2007. p. 145-73.

[33] Tsao TC, Juang YC, Lan RS, Shieh WB, Lee CH. Respiratory failure of acute organophosphate and carbamate poisoning. Chest 1990;98:631-6.

[34] Karki P, Ansari JA, Bhandary S, Koirala S. Cardiac and electrocardiographical manifestations of acute organophosphate poisoning. Singapore Med J 2004;45:385-9.

[35] Ludomirsky A, Klein HO, Sarelli P, Becker B, Hoffman S, Taitelman U, et al. Q-T prolongation and polymorphous ("torsade de pointes") ventricular arrhythmias associated with organophosphorus insecticide poisoning. Am J Cardiol 1982;49:1654-8.

[36] Abraham S, Oz N, Sahar R, Kadar T. QTc prolongation and cardiac lesions following acute organophosphate poisoning in rats. Proc West Pharmacol Soc 2001;44:185-6.

[37] Abraham S, Rabinovitz I, Oz N. Acute organophosphate poisoning sensitizes the rat myocardium to the arrhythmogenic effect of epinephrine. Pharamacologist 2002;44 (Suppl.):A14.

[38] Allon N, Rabinovitz I, Manistersky E, Weissman BA, Grauer E. Acute and long-lasting cardiac changes following a single whole-body exposure to sarin vapor in rats. Toxicol Sci 2005;87:385-90.

[39] Chuang FR, Jang SW, Lin JL, Chern MS, Chen JB, Hsu KT. QTe prolongation indicates a poor prognosis in patients with organophosphate poisoning. Am J Emerg Med 1996;14:451-3. 
[40] James JT, Manthei JH, Goodwin BS, Heitkamp D, Liebenberg SP. Clinical chemistry reference values in two breeds of swine and their changes during percutaneous exposure to soman. Am J Vet Res 1987;48:284-8.

[41] Diercks DB, Shumaik GM, Harrigan RA, Brady WJ, Chan TC. Electrocardiographic manifestations: electrolyte abnormalities. J Emerg Med 2004;27:153-60. 


\section{Legends for Figures}

Figure 1. Circulating BChE (panel A) and AChE (panel B) levels in the blood of minipigs injected with saline or $\mathrm{Hu}$ BChE and exposed to GB vapor. All values are mean \pm S.E.M. Symbols represent the following pretreatments prior to exposure to GB vapor: ( $\mathbf{\square})$, saline; ( $\mathbf{\Delta})$, $7.5 \mathrm{mg} / \mathrm{kg}$ of $\mathrm{Hu} \mathrm{BChE}$.

Figure 2. Regenerated GB in RBCs and plasma from saline- and Hu BChE-pretreated minipigs following exposure to GB vapor. Values are shown for venous blood taken from animals pretreated with saline at 10 min post-GB exposure (the last sample collected before death of the animal) or $\mathrm{Hu} \mathrm{BChE}(7.5 \mathrm{mg} / \mathrm{kg})$ at $60 \mathrm{~min}$ post-GB exposure. All values are mean \pm S.E.M. Blood was separated into erythrocyte (solid bars) and plasma (hatched bars) fractions by centrifugation and GB was regenerated from 0.1-0.25 $\mathrm{g}$ of each sample as described [29].

Figure 3. HR, PR, and QT intervals for saline- and Hu BChE-pretreated minipigs exposed to GB vapor. Göttingen minipigs were treated with saline or $\mathrm{Hu} \mathrm{BChE} 24 \mathrm{~h}$ prior to exposure to air or $11.4 \mathrm{mg} / \mathrm{m}^{3} \mathrm{~GB}$ vapor for $10 \mathrm{~min}$. HR (A), PR (B), and QT (C) intervals were analyzed as a function of time relative to death. Symbols represent the following pretreatments prior to exposure to GB vapor: (匹), saline; $(\boldsymbol{\Delta}), 7.5 \mathrm{mg} / \mathrm{kg}$ of $\mathrm{Hu} \mathrm{BChE}$, while ( $\square$ ) represents exposure to air. * indicates that treatment as a function of time is significantly different from saline-injected, air-exposed control animals.

Figure 4. HR, PR, and QT1c intervals for Hu BChE-pretreated minipigs 1 week after exposure to GB vapor. HR (A), PR (B) and QT1c intervals (C) for minipigs pretreated with 7.5 
$\mathrm{mg} / \mathrm{kg}$ of $\mathrm{Hu} \mathrm{BChE}$ are indistinguishable from tracings taken from the time of exposure. Symbols represent the following: $(\boldsymbol{\Delta})$, pretreatment with $7.5 \mathrm{mg} / \mathrm{kg}$ prior to exposure to GB vapor; ( $\square)$, exposure to air.

Figure 5. 3D plots of EEG power spectra as a function of time from onset of GB vapor exposure. EEG spectral analysis of minipigs pretreated with saline (A, B) or $7.5 \mathrm{mg} / \mathrm{kg}$ of $\mathrm{Hu}$ BChE (C) and exposed to air (A) or GB vapor (B and C) are shown. Although there is an increase in delta band power in GB-exposed pigs, peak voltage is indistinguishable from airexposed controls. EEG spectra for minipigs in panel C 1 week after exposure are shown in panel D. 
Table 1. Effect of Hu BChE pretreatment on key blood chemistry parameters in minipigs exposed to GB vapor

\begin{tabular}{|c|c|c|c|c|c|c|}
\hline \multirow{2}{*}{ Parameter } & \multicolumn{2}{|c|}{$\operatorname{Air}^{\mathrm{a}}$} & \multicolumn{2}{|c|}{ Saline + GB } & \multicolumn{2}{|c|}{$7.5 \mathrm{mg} / \mathrm{kg} \mathrm{Hu} \mathrm{BChE}+\mathrm{GB}$} \\
\hline & Baseline & Terminal & Baseline & Terminal & Baseline & Terminal \\
\hline Glucose (mg/dl) & $101 \pm 7$ & $106 \pm 5$ & $115 \pm 3$ & $156 \pm 0$ & $118 \pm 8$ & $108 \pm 6$ \\
\hline $\mathrm{Na}^{+}(\mathrm{mmol} / \mathrm{l})$ & $140 \pm 1$ & $139 \pm 1$ & $139 \pm 2$ & $140 \pm 1$ & $138 \pm 1$ & $141 \pm 1$ \\
\hline $\mathrm{K}^{+}(\mathrm{mmol} / \mathrm{l})$ & $4.3 \pm 0.3$ & $4.2 \pm 0.1$ & $4.3 \pm 0.2$ & $4.9 \pm 0.5$ & $4.3 \pm 0.03$ & $4.2 \pm 0.4$ \\
\hline $\operatorname{HCT}(\%)$ & $34.0 \pm 1.0$ & $30.7 \pm 1.0$ & $25.5 \pm 7.5$ & $31.5 \pm 3.5$ & $27.0 \pm 4.0$ & $32.5 \pm 0.5$ \\
\hline $\mathrm{Hb}(\mathrm{mg} / \mathrm{dl})$ & $11.1 \pm 0.3$ & $10.4 \pm 0.3$ & $8.7 \pm 2.5$ & $10.7 \pm 1.2$ & $9.2 \pm 1.4$ & $9.5 \pm 0.9$ \\
\hline $\mathrm{pH}$ & $7.38 \pm 0.03$ & $7.42 \pm 0.01$ & $7.39 \pm 0.005$ & $6.89 \pm 0$ & $7.41 \pm 0.02$ & $7.43 \pm 0.04$ \\
\hline $\mathrm{pCO}_{2}(\mathrm{mmHg})$ & $48.6 \pm 1.1$ & $49.7 \pm 1.5$ & $46.2 \pm 7.5$ & $115.9 \pm 0$ & $41.9 \pm 5.3$ & $48.0 \pm 1.4$ \\
\hline $\mathrm{pO}_{2}(\mathrm{mmHg})$ & $37.7 \pm 1.9$ & $37.4 \pm 1.2$ & $38.5 \pm 0.5$ & $18.0 \pm 0$ & $38.5 \pm 1.7$ & $38.0 \pm 1.0$ \\
\hline $\mathrm{T} \mathrm{CO}_{2}(\mathrm{mmol} / \mathrm{l})$ & $32.5 \pm 1.3$ & $34.0 \pm 1.3$ & $30.5 \pm 6.5$ & $26 \pm 0$ & $28.3 \pm 4.5$ & $34.0 \pm 4.0$ \\
\hline $\mathrm{HCO}_{3}^{-}(\mathrm{mmol} / \mathrm{l})$ & $30.9 \pm 1.4$ & $32.5 \pm 0.9$ & $29.4 \pm 6.1$ & $22.3 \pm 0$ & $28.3 \pm 3.5$ & $32.4 \pm 3.9$ \\
\hline $\mathrm{sO}_{2}(\%)$ & $74.8 \pm 1.3$ & $71.0 \pm 1.3$ & $72.5 \pm 0.5$ & $11 \pm 0$ & $73.3 \pm 2.7$ & $72.3 \pm 1.5$ \\
\hline
\end{tabular}

All values are mean \pm S.E.M. Baseline values were measured from venous blood taken before air or GB vapor exposure commenced and terminal values were measured from venous blood taken at $60 \mathrm{~min}$ post-GB (10 min GB $+50 \mathrm{~min}$ air) or air exposure, or from the last sample collected before death of the animal. As saline-treated animals $(n=3)$ died at 10-23 min of GB vapor exposure, the last blood was drawn at $4 \min (n=1)$ or $10 \min (n=1)$; blood from the third animal could not be drawn due to the blockage of the catheter. Abbreviations are described in the methods section. ${ }^{\mathrm{a}}$ Data from [24]. 

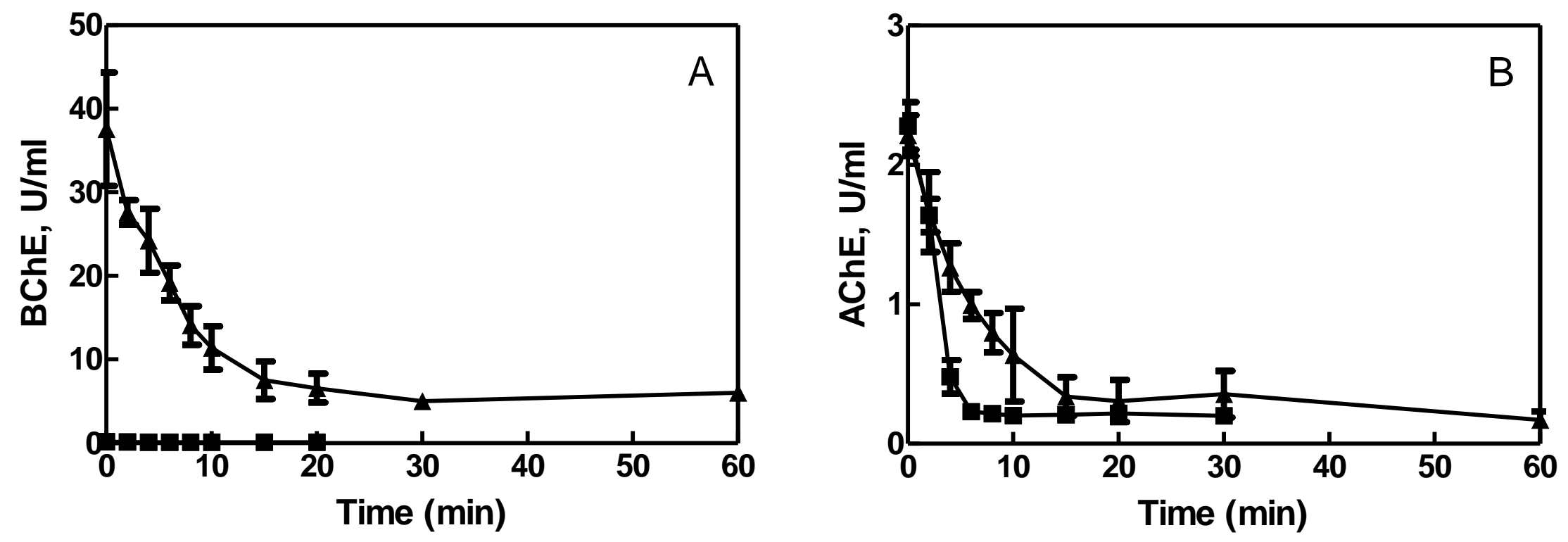


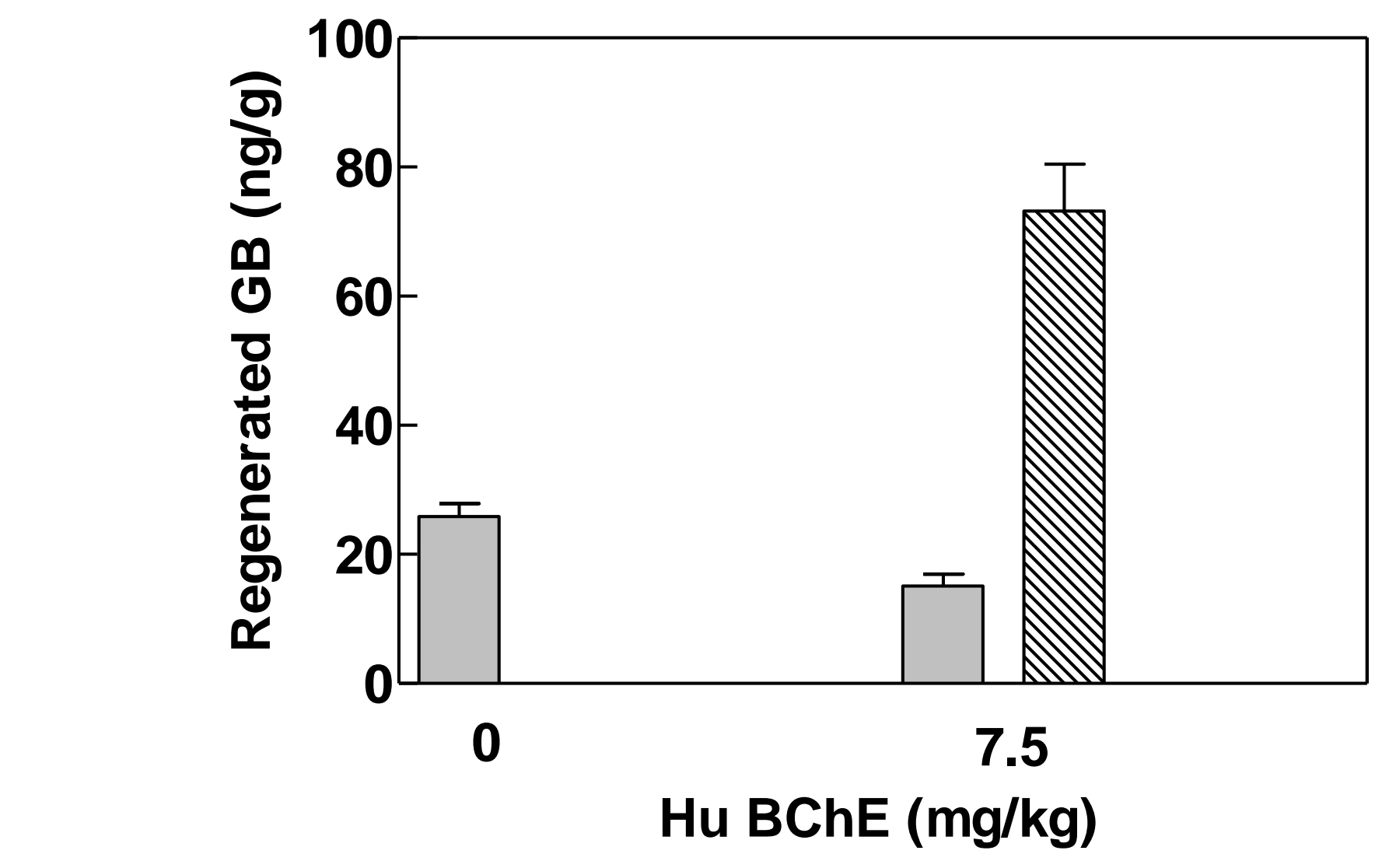

Figure 2

Hu BChE (mg/kg)

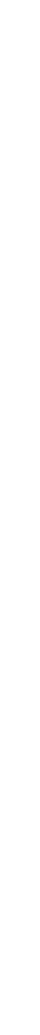



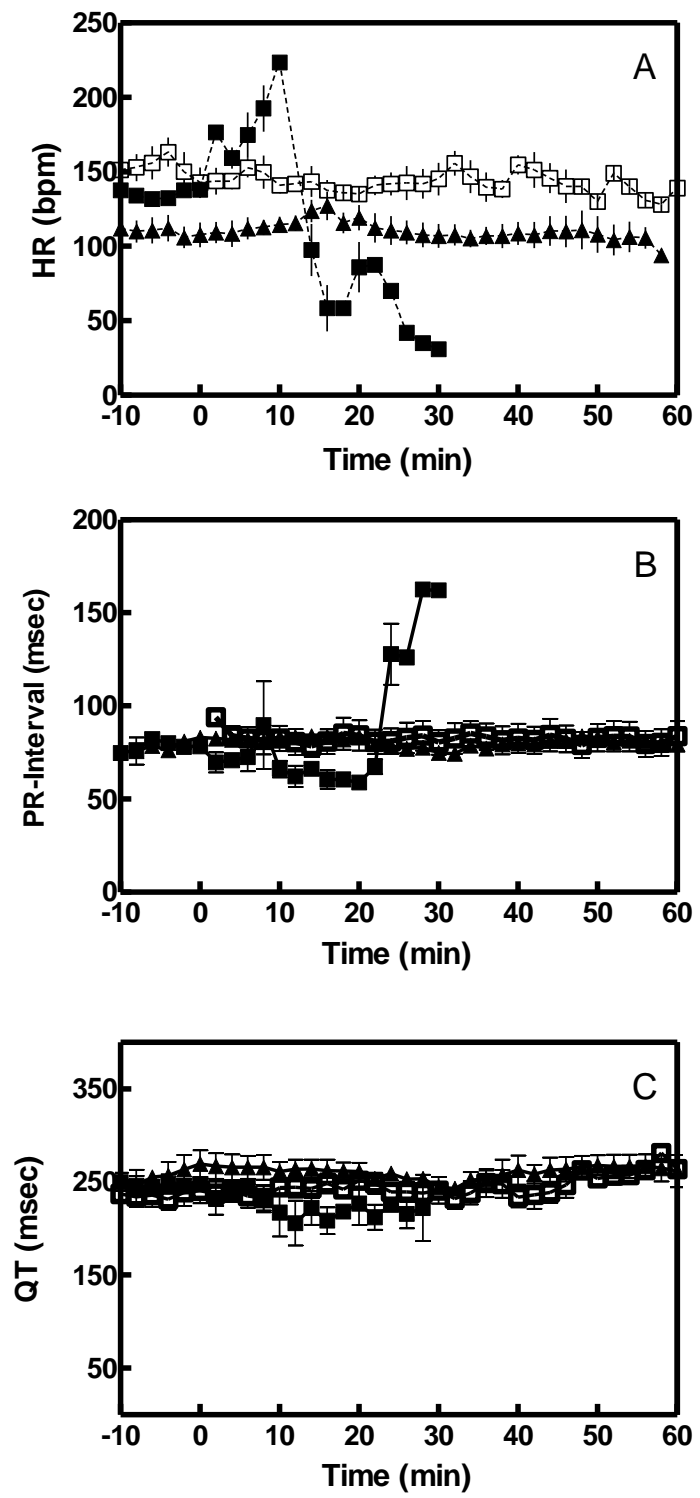
Figure 4
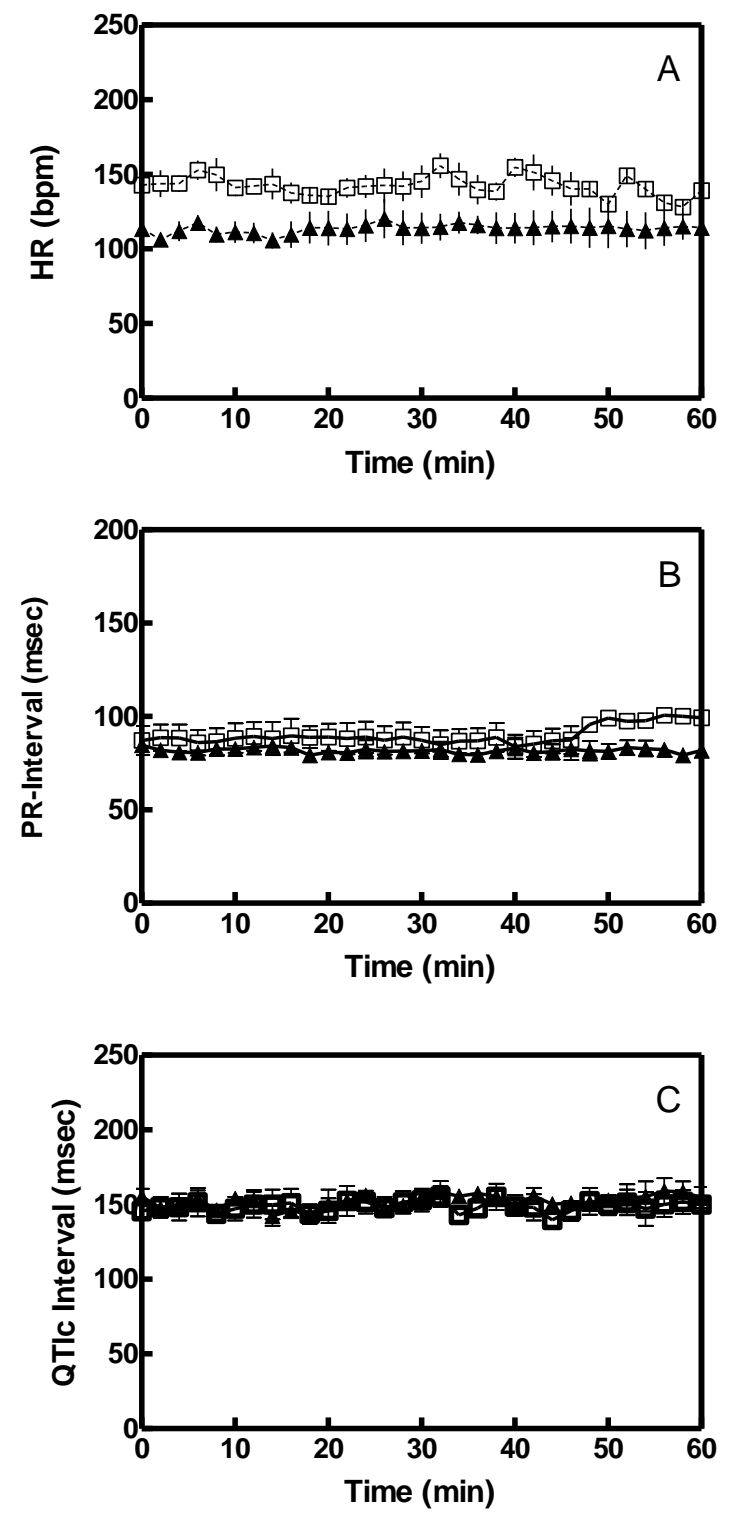


\section{Fiqube 5}
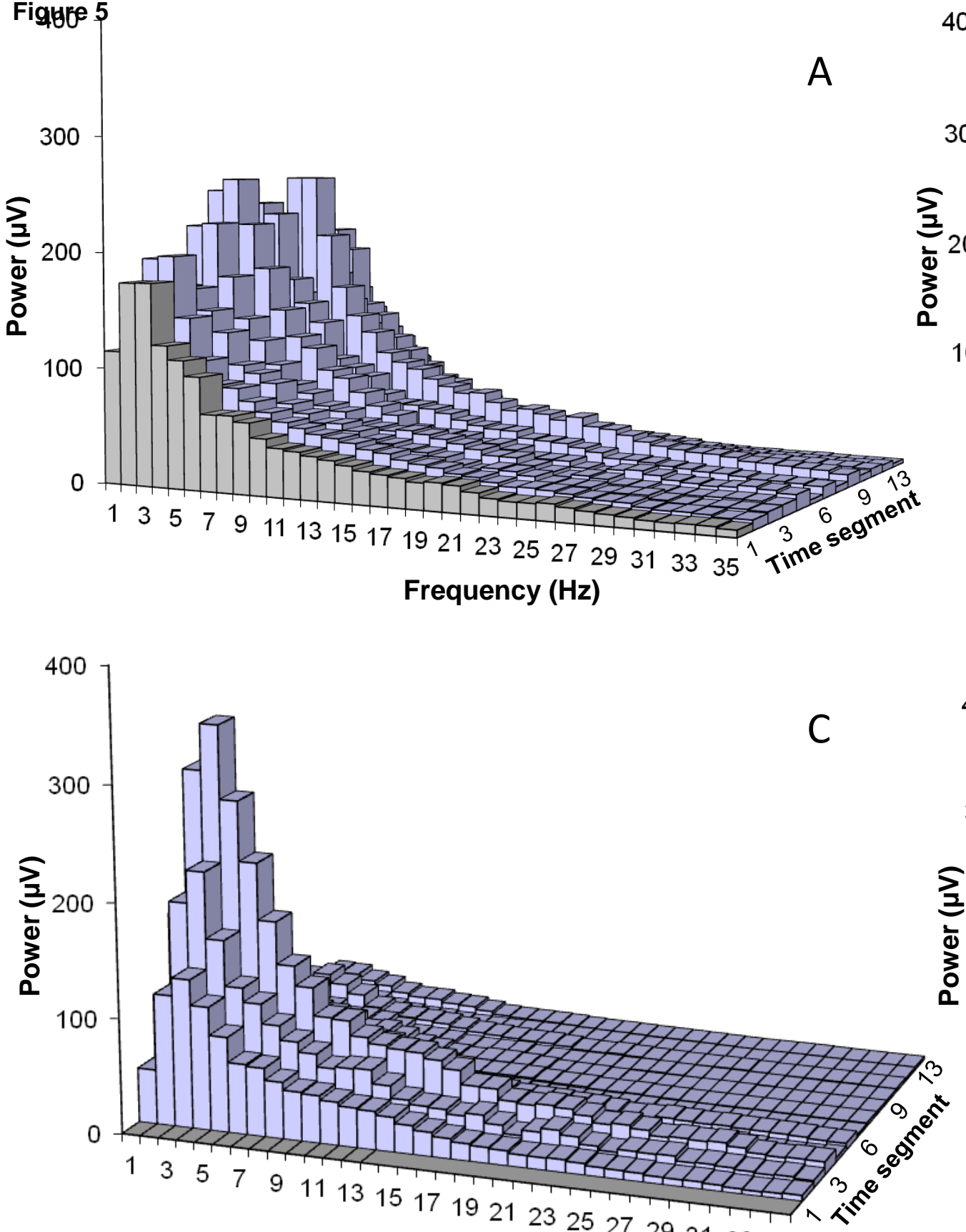

Frequency $(\mathrm{Hz})$
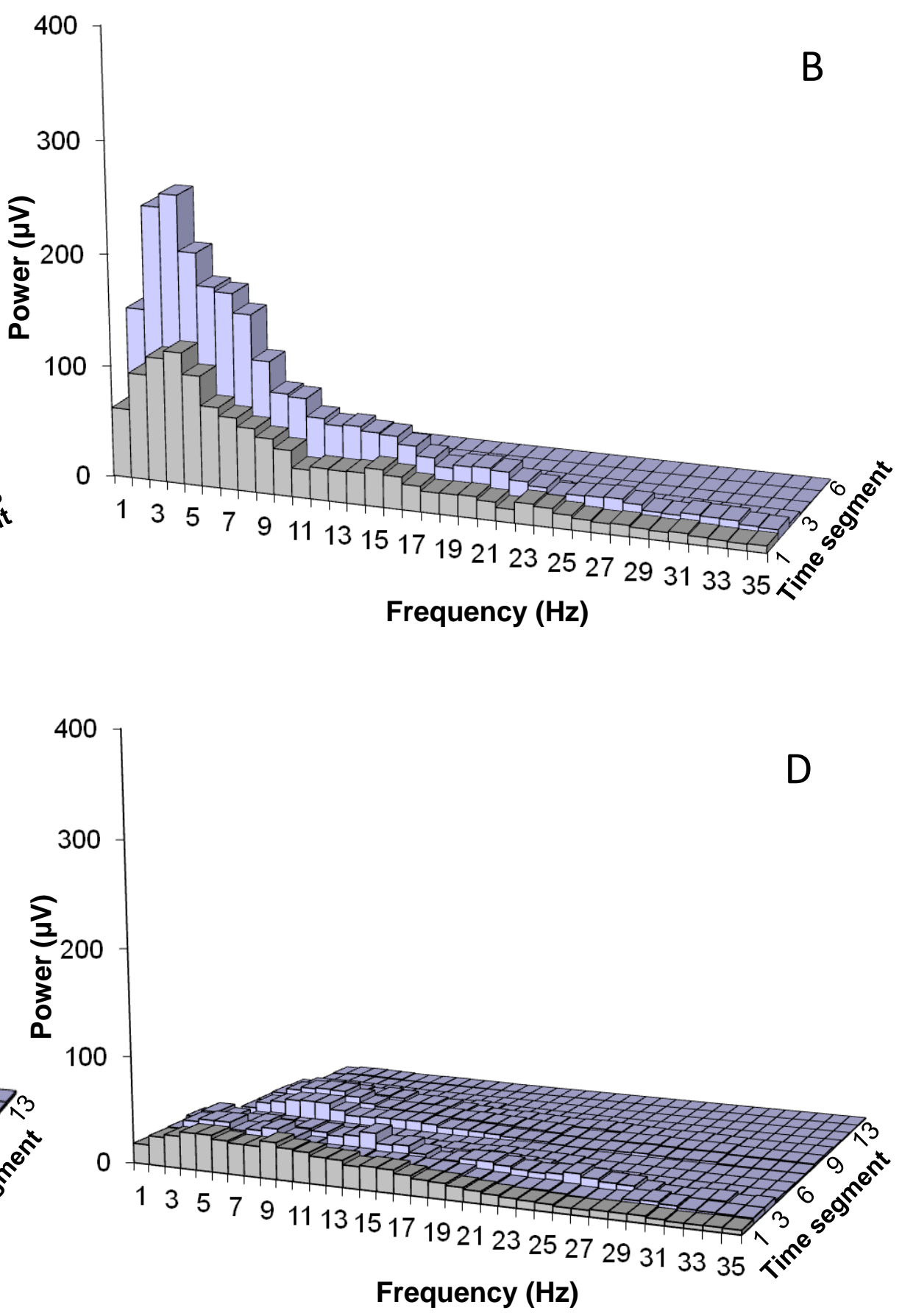